\title{
Mutual Relation between Distributions Based on Number and Mass in The Particle Size-Shape Dispersion Diagram
}

\author{
Takashi Itoh and Yoshimoto Wanibe \\ Department of Materials Processing Engineering, Graduate School of Engineering, Nagoya University, Furo-cho, Chikusa-ku, \\ Nagoya 464-8603.
}

Received December 1, 1997

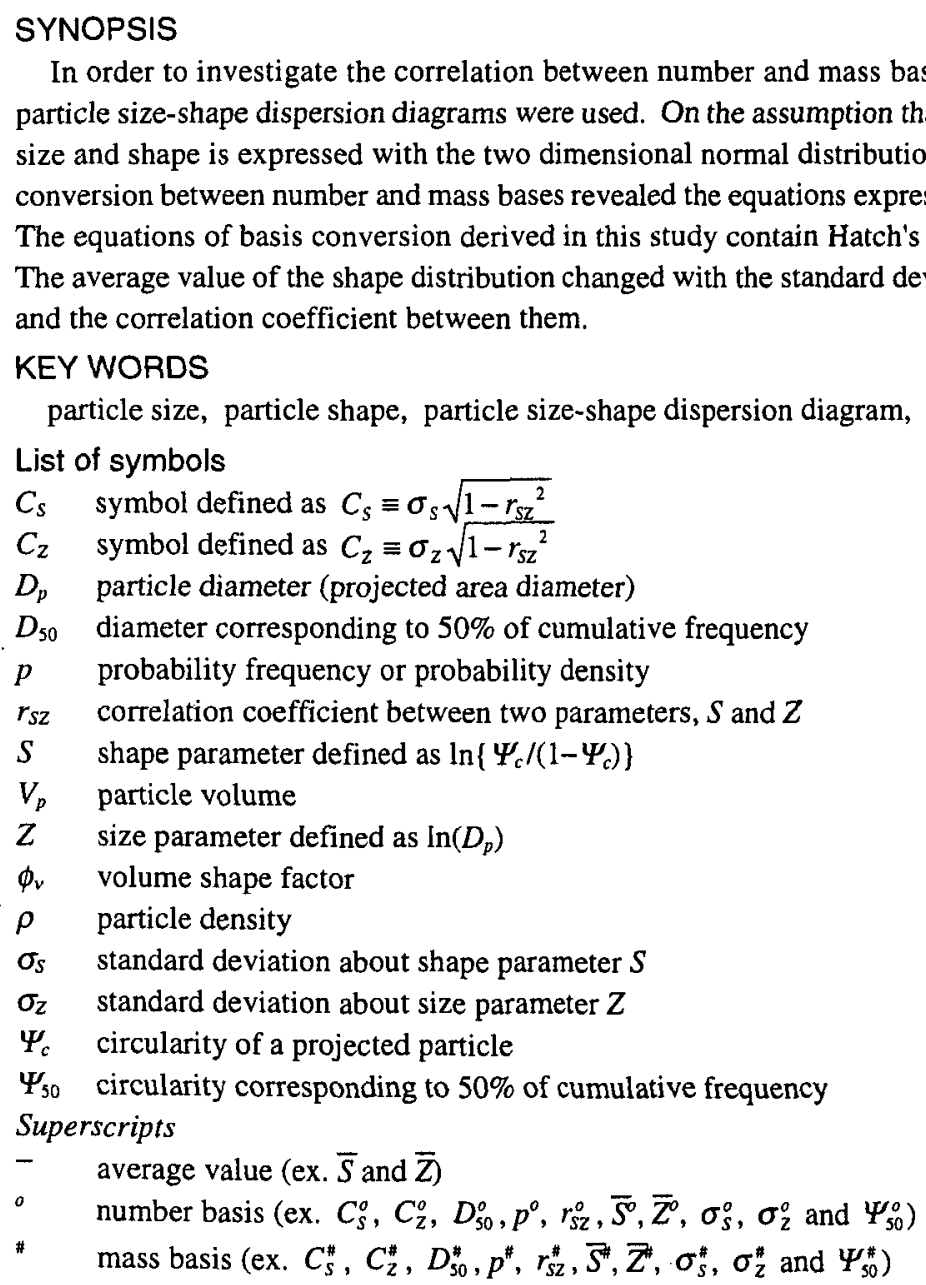

\section{Introduction}

Particle size distribution has been determined as a powder property in vast powder industry. There can be various particle size distributions based on different measuring methods selected ${ }^{13}$. Most popular ones are the number- and mass-based distributions in particle size. Then mutual relation between the two distributions is necessary for conversion needed in comparison among the ones measured on the different bases, and/or in consideration for the designing of blending the powders that have the size distributions given on the number basis.

Two distribution laws of log-normal and Rosin-Rammler have been widely used for expressing the particle size distribution. In the log-normal distribution law, the relationship between the number and mass bases has been expressed by Hatch's equations derived analytically ${ }^{2}$. The similar relation in the Rosin-Rammler distribution law has been reported in the previous work ${ }^{3}$. The relations in these 
laws can be valid under the assumption that all particles in a powder are of a same shape. In actual powder, however, there is a particle shape distribution. Then the above assumption has no reality.

In the present work, the mutual correspondence between the number- and mass-based distributions is discussed by using some correlation between the size and shape distributions in the particle size-shape dispersion diagram proposed for more precise characterization ${ }^{4-9}$. It has been proposed that the dispersion condition between particle size and shape in the diagram can be expressed with a twodimensional normal distribution law. The theoretical conversion between the number and mass bases in the above diagram is studied and the conversing equations are derived. The results of the basis conversion are also shown by changes in the dispersion diagram.

\section{Particle size-shape dispersion diagram and two- dimensional normal distribution law}

The previous reports have already shown the characteristics of the particle size-shape dispersion diagram ${ }^{4}$, and some quantitative relations between the characterized powder properties and the behavior, e.g. the packing density ${ }^{5-8)}$ or the fluidity ${ }^{9}$. Here the diagram and the two-dimensional normal distribution law are briefly summarized.

An example of the particle size-shape dispersion diagram is shown in Fig. 1. The axes of abscissas and ordinates are expressed with the particle diameter $D_{p}$ (projected area diameter) and the circularity $\Psi_{c}$ that is one of the shape indexes, respectively. This diagram is composed of the size frequency distribution (A), the shape frequency distribution (B) and the two-dimensional figure (C), indicating the dispersion condition between the size and shape. The two-dimensional figure in the diagram made on the number basis is usually characterized by plotting the cross points which correspond to the coordinates of size and shape in the powder particles. It can also be expressed with the iso-probability density curves as shown in Fig. 1. The particle size-shape dispersion diagram can be used for numerical characterization of powder characteristics according to the distributions of particle size and shape and the correlation between them.

The axis of particle size in the diagram is on a logarithmic scale, that is $\ln \left(D_{p}\right)$, and the other axis of particle shape is scaled with $\ln \left\{\Psi_{c} /\left(1-\Psi_{c}\right)\right\}$. These styles of axes are based on the condition that the size and shape distributions obey the normal distribution laws having the parameters $\ln \left(D_{p}\right)$ and $\ln \left\{\Psi_{c} /\left(1-\Psi_{c}\right)\right\}$, respectively ${ }^{4)}$. Thus the dispersion figure in this diagram is an ideal condition that obeys the two-dimensional normal distribution law on the number basis. In this case the number-based size distribution (A) is expressed by the following equation,

$$
p^{\circ}(Z)=\frac{1}{\sqrt{2 \pi} \sigma_{z}^{\circ}} \exp \left\{-\frac{\left(Z-\bar{Z}^{\circ}\right)^{2}}{2 \sigma_{z}^{0^{2}}}\right\},
$$

where $p^{\circ}(Z)$ is the probability frequency, $Z$ the size parameter defined as $Z \equiv \ln \left(D_{p}\right), \sigma_{Z}^{o}$ the standard deviation about parameter $Z$, and $\bar{Z}$ the average value of parameter $Z$ or the logarithmic value of the diameter corresponding to $50 \%$ of cumulative frequency, $D_{50}^{o}$. The particle shape distribution of number basis (B) is similarly expressed as follows:

$$
p^{o}(S)=\frac{1}{\sqrt{2 \pi} \sigma_{s}^{o}} \exp \left\{-\frac{\left(S-\bar{S}^{o}\right)^{2}}{2 \sigma_{s}^{o^{2}}}\right\} .
$$

The symbols $p^{o}(S), \sigma_{s}^{o}$ and $\bar{S}^{o}$ are the probability frequency, the standard deviation, and the average value about the shape parameter $S$ defined as $S \equiv \ln \left\{\Psi_{c} /\left(1-\Psi_{c}\right)\right\}$, respectively. $\bar{S}^{o}$ can be defined as $\bar{S}^{o} \equiv \ln \left\{\Psi_{s_{0}}^{o} /\left(1-\Psi_{s_{0}}^{o}\right)\right\}$, where $\Psi_{s 0}^{\circ}$ is the circularity corresponding to $50 \%$ of cumulative frequency. Furthermore, the number-based dispersion figure $(C)$ between the size and shape distributions is expressed with the two-dimensional normal distribution law,

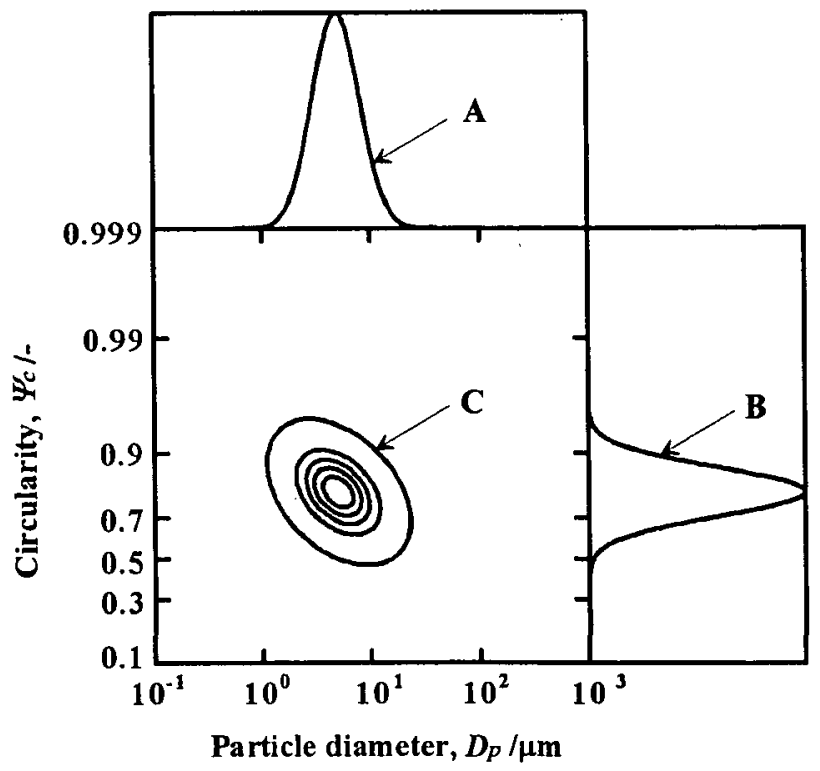

Fig.1 Particle size-shape dispersion diagram based on the twodimensional normal distribution law; symbols A, B and C indicate the size frequency distribution, the shape frequency one and the two-dimensional figure indicating the dispersion condition between size and shape, respectively.

( $\left.D_{50}^{o}=5 \mu \mathrm{m}, \sigma_{Z}^{o}=0.5, \Psi_{s 0}^{o}=0.8, \sigma_{s}^{o}=0.5, r_{s Z}^{o}=-0.4\right)$ 


$$
\begin{aligned}
& p^{o}(S, Z)=\frac{\sqrt{1-r_{s z}^{o}}}{2 \pi C_{s}^{o} C_{z}^{o}} \exp \\
& {\left[-\frac{1}{2}\left\{\frac{\left(S-\bar{S}^{o}\right)^{2}}{C_{s}^{o^{2}}}-2 r_{s z}^{o} \frac{\left(S-\bar{S}^{o}\right)\left(Z-\bar{Z}^{o}\right)}{C_{S}^{o} C_{z}^{o}}+\frac{\left(Z-\bar{Z}^{o}\right)^{2}}{C_{z}^{o^{2}}}\right\}\right]}
\end{aligned}
$$

where $p^{\circ}(S, Z)$ and $r_{s z}^{o}$ are the probability density and the correlation coefficient between the two parameters, $S$ and $Z$. The symbols $C_{s}^{o}$ and $C_{z}^{o}$ are defined as $C_{s}^{o} \equiv \sigma_{s}^{o} \sqrt{1-r_{s z}^{o}}$ and $C_{z}^{o} \equiv \sigma_{z}^{o} \sqrt{1-r_{s z}^{o}}$.

There are three types of diagram according to the correlation coefficient $r_{s z}^{o}$. The examples are indicated in Fig. 2 showing iso-probability curves with correlation coefficients of negative, zero, and positive values, respectively. Under the condition that the correlation coefficient has a negative value $\left(r_{s z}^{\circ}=-0.4\right)$ as shown in Fig.2(a), the powder has a tendency for the irregularity of the particle shape to increase with the particle size. Many powders are classified into this type. Fig.2(b) indicates the condition when $r_{s z}^{\circ}=0$. There is no correlation between size and shape under this condition, i.e. both distributions are independent of each other. The condition indicated in Fig.2(c) is another one, that is, the correlation coefficient has a positive value $\left(r_{s z}^{\circ}=0.4\right)$. In this case the powder has an opposite tendency to that shown in Fig.2(a).

\section{Derivations of Hatch's equations on the size distribution and basis conversion equation on the size-shape dispersion diagram}

Hatch's equations are well known as the conversion equations between number and mass bases of the particle size distribution that obeys the log-normal distribution law. Its theoretical derivation is summarized as follows:

The mass-based probability frequency $p^{*}$ of the particle size distribution is generally connected with the frequency $p^{o}$ on the number basis according to the following equation,

$$
p^{*} \equiv \frac{\rho \cdot \phi_{v} \cdot D_{p}^{3} \cdot p^{o}}{\int_{D_{p=0}}^{0}\left(\rho \cdot \phi_{v} \cdot D_{p}^{3} \cdot p^{0}\right) \mathrm{d} Z} .
$$

This equation is obtained by normalizing the product between the number-based probability frequency $p^{o}$ and the mass of a particle $\rho \cdot \phi_{v} \cdot D_{p}{ }^{3}$ with its integration. The symbols $\rho$ and $\phi_{v}$ are the particle density and the volume shape factor, respectively. Assuming particles of a same density and a similar shape, Eq. (4) can be simplified into the equation,

$$
p^{*} \equiv \frac{D_{p}^{3} \cdot p^{0}}{\int_{D_{p=0}}^{\infty}\left(D_{p}^{3} \cdot p^{0}\right) \mathrm{d} Z}
$$
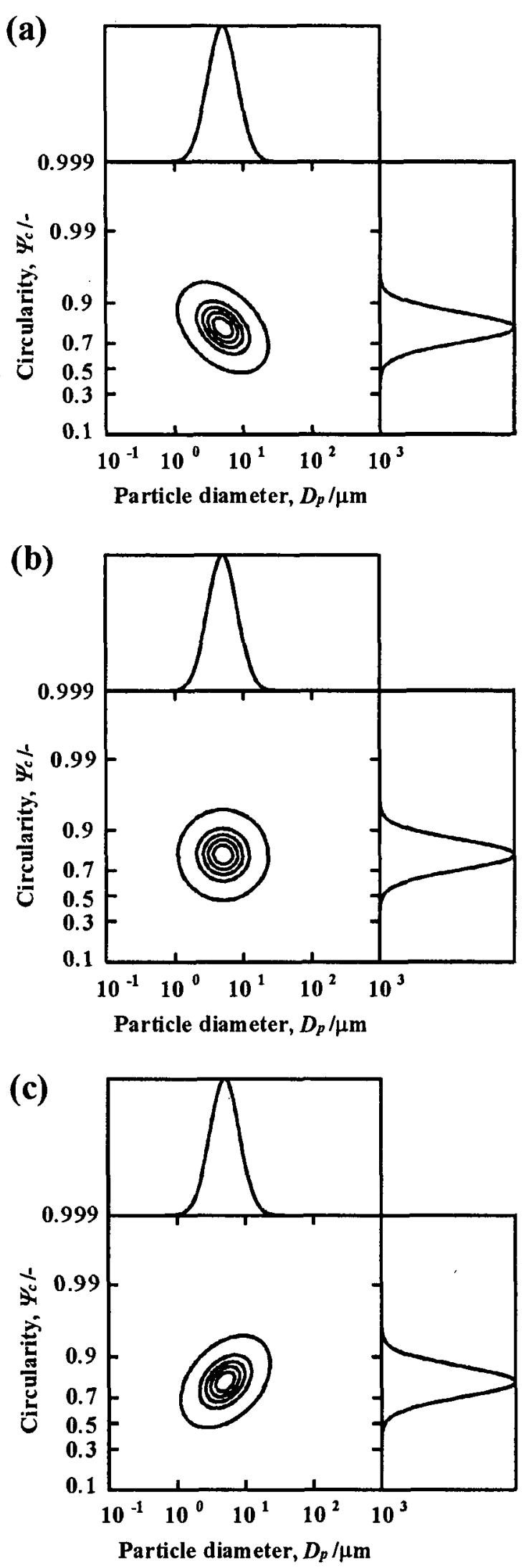

Fig.2 Typical particle size-shape dispersion diagrams under three different conditions: (a) $r_{S Z}^{o}=-0.4$; (b) 0 ; and (c) 0.4 .

$\left(D_{50}^{o}=5 \mu \mathrm{m}, \sigma_{z}^{o}=0.5, \Psi_{s 0}^{o}=0.8, \sigma_{s}^{o}=0.5\right.$ ) 
For the probability frequency $p^{0}$ of the number-based size distribution expressed with Eq. (1), the above equation becomes

$$
p^{*}(Z)=\frac{\frac{1}{\sqrt{2 \pi} \sigma_{z}^{o}} \exp \left\{-\frac{\left(Z-\bar{Z}^{o}\right)^{2}}{2 \sigma_{z}^{o^{2}}}+3 Z\right\}}{\int_{-\infty}^{\infty} \frac{1}{\sqrt{2 \pi} \sigma_{z}^{o}} \exp \left\{-\frac{\left(Z-\bar{Z}^{o}\right)^{2}}{2 \sigma_{z}^{o^{2}}}+3 Z\right\} d Z} .
$$

After arrangement of the above equation,

$$
p^{\prime \prime}(Z)=\frac{\frac{D_{s 0}^{o^{3}}}{\sqrt{2 \pi} \sigma_{z}^{o}} \exp \left(\frac{9}{2} \sigma_{z}^{o^{2}}\right) \exp \left\{-\frac{\left(Z-\bar{Z}^{o}-3 \sigma_{z}^{o^{2}}\right)^{2}}{2 \sigma_{z}^{o^{2}}}\right\}}{\int_{-\infty}^{\infty} \frac{D_{s 0}^{o^{3}}}{\sqrt{2 \pi} \sigma_{z}^{o}} \exp \left(\frac{9}{2} \sigma_{z}^{o^{2}}\right) \exp \left\{-\frac{\left(Z-\bar{Z}^{o}-3 \sigma_{z}^{o^{2}}\right)^{2}}{2 \sigma_{z}^{o^{2}}}\right\} \mathrm{d} Z}
$$

is obtained. This equation is simplified as follows:

$$
p^{\sharp}(Z)=\frac{\frac{1}{\sqrt{2 \pi} \sigma_{z}^{o}} \exp \left\{-\frac{\left(Z-\bar{Z}^{\circ}-3 \sigma_{z}^{o^{2}}\right)^{2}}{2 \sigma_{z}^{o^{2}}}\right\}}{\int_{-\infty}^{\infty} \frac{1}{\sqrt{2 \pi} \sigma_{z}^{\circ}} \exp \left(\frac{9}{2} \sigma_{z}^{o^{2}}\right) \exp \left\{-\frac{\left(Z-\bar{Z}^{o}-3 \sigma_{z}^{o^{2}}\right)^{2}}{2 \sigma_{z}^{o^{2}}}\right\} \mathrm{d} Z} .
$$

Since the denominator in this equation is infinite integral of the normal distribution that has the average value $\bar{Z}^{\circ}+3 \sigma_{z}^{\circ 2}$ and the standard deviation $\sigma_{z}^{o}$, its value becomes unity. The probability frequency $p^{\#}$ of the mass-based size distribution becomes

$$
p^{*}(Z)=\frac{1}{\sqrt{2 \pi} \sigma_{z}^{\circ}} \exp \left\{-\frac{\left(Z-\bar{Z}^{o}-3 \sigma_{z}^{o^{2}}\right)^{2}}{2 \sigma_{z}^{o^{2}}}\right\}
$$

By comparing Eqs. (1) and (9), it is clear that the converted distribution equation of mass basis has the same style with the number-based distribution obeying normal distribution law. Then we have the following basis conversion equations:

$$
\bar{Z}^{*}=\bar{Z}^{\circ}+3 \sigma_{z}^{o} \text { and } \sigma_{z}^{*}=\sigma_{z}^{o}
$$

or

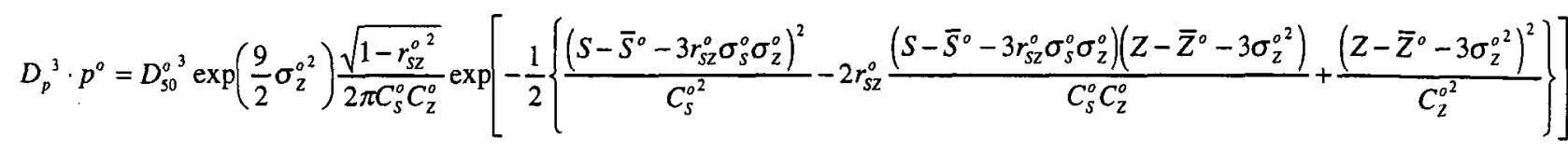


The above equation contains the two-dimensional normal distribution that has the two average values $\bar{S}^{\circ}+3 r_{s z}^{o} \sigma_{s}^{o} \sigma_{z}^{o}$ and $\bar{Z}^{\circ}+3 \sigma_{z}^{\circ 2}$, the two standard deviations $\sigma_{s}^{\circ}$ and $\sigma_{z}^{\circ}$, and the correlation coefficient $r_{s z}^{o}$. The denominator in Eq. (12) corresponds to the double integral of Ep. (14). Since the infinite integral of the two-dimensional normal distribution becomes unity, the denominator in Eq. (12) can be expressed with the following equation.

$$
\int_{D_{p}=0}^{\infty} \int_{c=0}^{1}\left(D_{p}^{3} \cdot p^{0}\right) \mathrm{d} S \cdot \mathrm{d} Z=D_{s 0}^{o^{3}} \exp \left(\frac{9}{2} \sigma_{Z}^{o^{2}}\right)
$$

The probability density $p^{*}$ of the mass-based dispersion diagram can be derived.

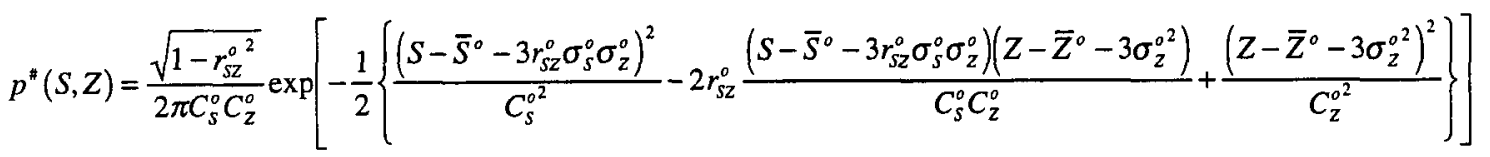
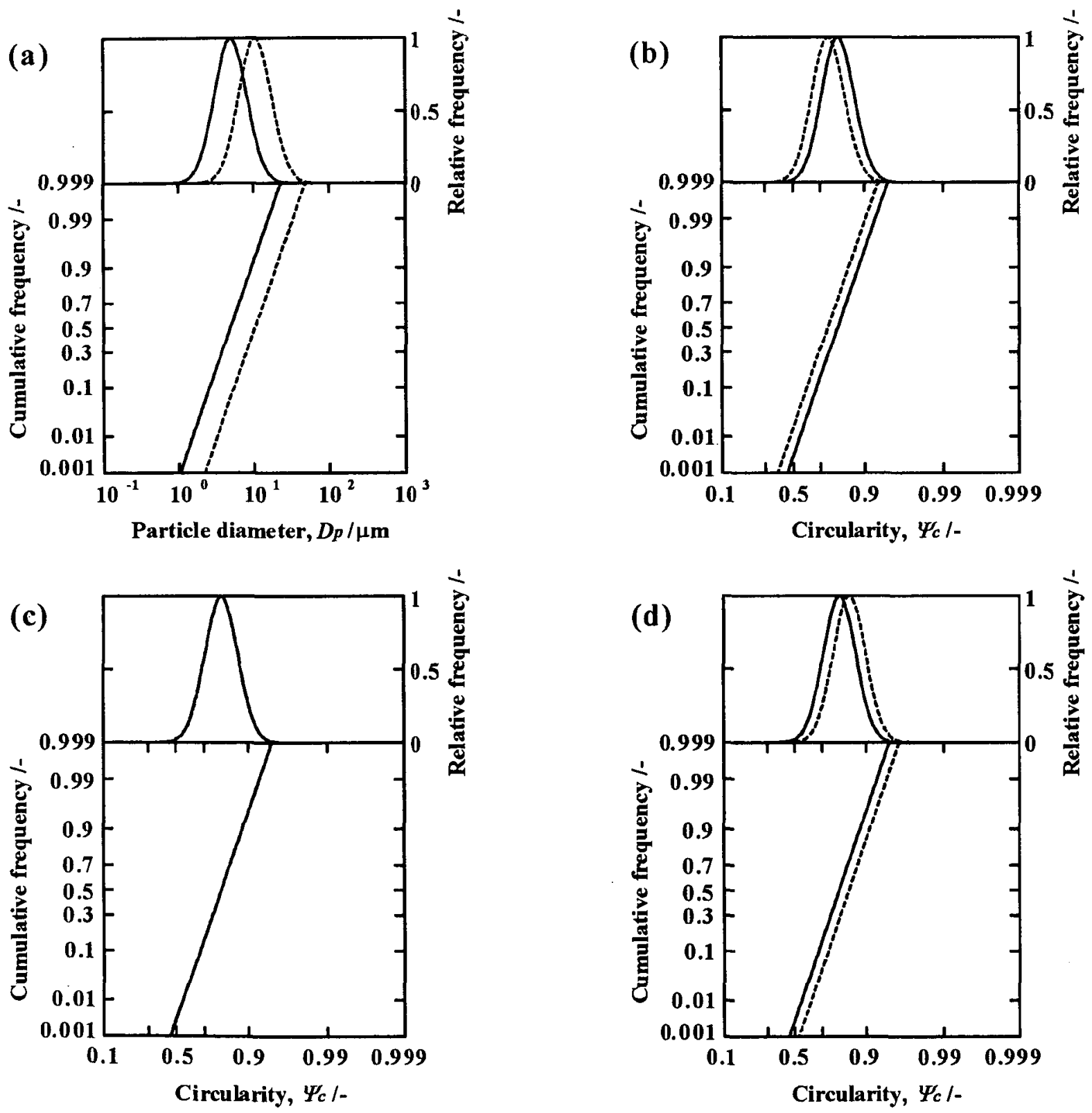

Fig.3 Relationship between number and mass bases on particle size and shape distributions under three different conditions of correlation coefficient: (a) shows particle size distribution and (b)-(d) show particle shape distributions of: (b) $r_{\mathrm{s}}^{o}=-0.4$; (c) 0 ; and (d) 0.4 , respectively.

$\left(D_{50}^{o}=5 \mu \mathrm{m}, \sigma_{2}^{o}=0.5, \Psi_{50}^{o}=0.8, \sigma_{s}^{o}=0.5\right)$ 
By comparing Eqs. (3) and (16), it is clear that the converted distribution equation of mass basis has the same style with the number-based one obeying two-dimensional normal distribution law. Then we have the following basis conversion equations;

$$
\begin{array}{ll}
\bar{S}^{*}=\bar{S}^{o}+3 r_{S Z}^{o} \sigma_{s}^{o} \sigma_{z}^{o}, & \sigma_{s}^{*}=\sigma_{s}^{o} \\
\bar{Z}^{*}=\bar{Z}^{o}+3 \sigma_{z}^{o}, & \sigma_{Z}^{*}=\sigma_{z}^{o} \\
\text { and } & r_{S Z}^{*}=r_{S Z}^{o}
\end{array}
$$

or

$$
\begin{array}{ll}
\frac{\Psi_{s 0}^{*}}{1-\Psi_{s 0}^{*}}=\frac{\Psi_{s 0}^{o}}{1-\Psi_{s 0}^{o}} \exp \left(3 r_{s z}^{o} \sigma_{s}^{o} \sigma_{z}^{o}\right), & \sigma_{s}^{*}=\sigma_{s}^{o} \\
D_{s 0}^{*}=D_{s 0}^{o} \cdot \exp \left(3 \sigma_{Z}^{o}\right), & \sigma_{Z}^{*}=\sigma_{Z}^{o} \\
\text { and } & r_{S Z}^{*}=r_{s Z}^{o}
\end{array}
$$

Various interesting information can be obtained from these equations. The basis conversion equations relating to the particle size distribution are in agreement with Hatch's equations. The correlation between the particle shape distributions (especially the average values of the distribution) of both bases, varies according to the standard deviations of both the size and shape distributions and the correlation coefficient indicating the dispersion condition. The standard deviation of the shape distribution and the correlation coefficient, are the same.

The cumulative and relative frequency distributions of particle size and shape on both bases are shown in Fig.3. The cumulative distributions are plotted on the normal probability diagrams. The relative frequency distribution is defined as the frequency distribution standardized by the maximum frequency. The solid and broken lines in these figures indicate the distributions based on number and mass, respectively. As for the particle size distribution shown in Fig.3(a), two straight lines, which are parallel in the cumulative frequency distribution diagram, indicates that the standard deviations of both the distributions have the same value. On the other hand, Fig.3(b),(c) and (d) show three types of particle shape distributions according to the correlation coefficient $r_{s z}^{o}$. In the case of $r_{s z}^{o}<0$ as shown in Fig.3(b), the broken straight line of mass basis slides from the solid straight line of number basis, toward the left side, that is, to a smaller average value of the shape distribution. In the case of no correlation $r_{s z}^{o}=0$ as shown in Fig.3(c), the mass-based shape distribution is in agreement with the number-based one. Fig.3(d) shows the opposite condition ( $r_{s z}^{o}>0$ ) to Fig.3(b). The broken straight line of the mass basis moves to a larger average value of the shape distribution. The standard deviations keep the same value in all cases.
The three types are also shown in Fig.4(a)-(c) of the particle size-shape dispersion diagrams. These figures indicates the results obtained under the same number-based conditions, except to the correlation coefficient $r_{s z}^{o}$, i.e. Fig.4(a) is the case of negative correlation $\left(r_{s z}^{o}=-0.4\right)$, (b) is the case of non-correlation $\left(r_{s z}^{o}=0\right)$, and (c) is the case of positive correlation. The solid and broken lines are respectively based on the number and mass bases the same as in Fig.3. The mutual relations between bases of the size distribution are the same in these three dispersion diagrams. It means that they don't depend on the correlation coefficient $r_{s z}^{o}$. In the conversion of number basis to mass basis, the dispersion condition between the size and shape in the diagram moves toward the right and down, the right, or the right and up, which depends upon the negative correlation, non-correlation, or positive correlation, respectively. This means that the dispersion condition is affected by the basis conversion.

The adequacy of Hatch's equations (10) can be easily confirmed by comparison between results of number- and mass-based size distribution analyses, e.g. microscopic method and sieve method. Since mass-based size-shape dispersion analysis has not been established yet, the adequacy of the basis conversion equations (17), which can be derived theoretically, can be hardly confirmed by measurement. The confirmation is possible as the classifying-shape technology develops. The theoretical process deriving the basis conversion equations (17), however, can not be used only for the size-shape dispersion diagram of powder, but also for the diagrams of all discrete materials, e.g. the short fiber which has been reported in the previous works ${ }^{10.11}$.

\section{Conclusion}

In this work the particle size-shape dispersion diagrams are used in order to investigate the correlation between number and mass bases according to both the particle size and shape. On the assumption that the dispersion condition between particle size and shape is expressed with the twodimensional normal distribution laws, the theoretical consideration of the conversion between number and mass bases reveals the equations expressing the correlation between both the bases. The variations on the dispersion diagram are also researched according to the conversion equations.

The results are summarized as follows:

1. The basis conversion equations with respect to the particle size distribution are consistent with Hatch's equations. The standard deviation of the distribution does not change with the conversion of number basis 

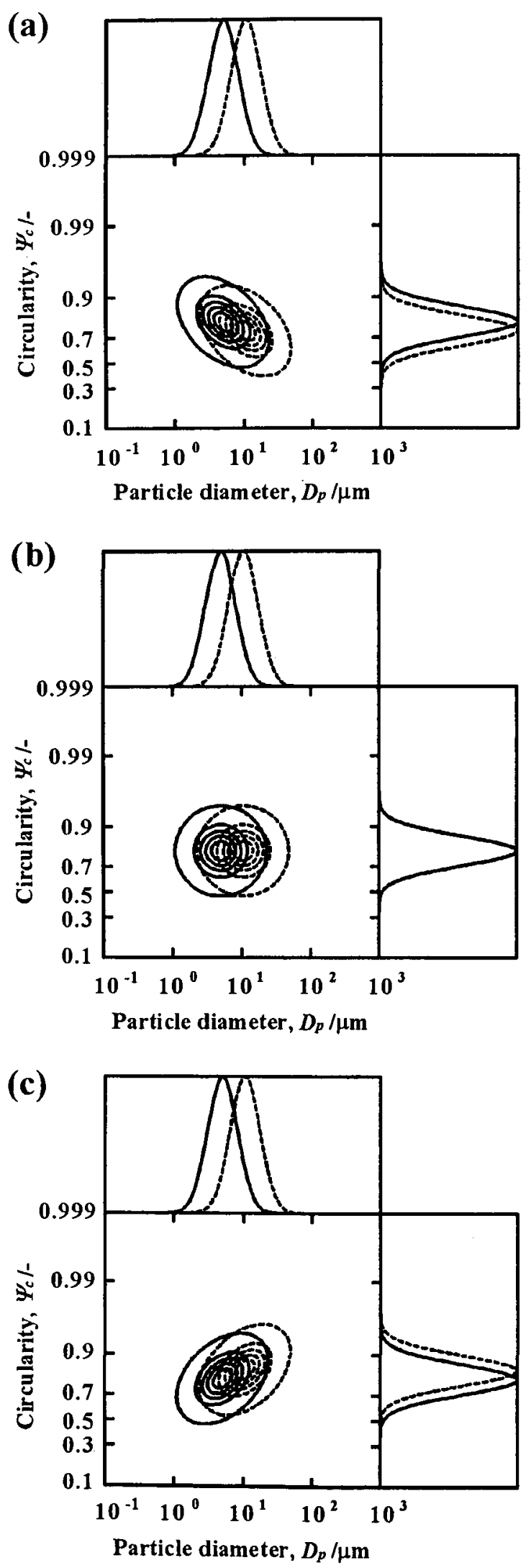

Fig.4 Three types of particle size-shape dispersion diagram based on both of number (solid line) and mass (broken line) bases: (a) $r_{S Z}^{o}=-0.4$; (b) 0 ; and (c) 0.4 .

$\left(D_{50}^{o}=5 \mu \mathrm{m}, \sigma_{Z}^{o}=0.5, \Psi_{s 0}^{o}=0.8, \sigma_{S}^{o}=0.5\right)$ into mass basis. On the other hand, the average value of the distribution increases with the conversion.

2. The standard deviation of the shape distribution does not changed with the conversion of number basis into mass basis the same as that of the size distribution. The average value of the distribution, however, changes with the standard deviations of both size and shape distributions and the correlation coefficient between them.

3. In the conversion of number basis to mass basis, the dispersion condition between the size and shape in the diagram moves toward the right down, the right and the right up according to the negative correlation, noncorrelation and positive correlation, respectively.

4. The theoretical process deriving the basis conversion equations can not be used only for the size-shape dispersion diagram of powder, but also for the diagrams of all discrete materials.

\section{References}

1) Ed. Soc. Powder Technology Jpn.: Huntai Kougaku Binran, Nikkan Kogyo Sinbunsya, (1986)15.

2) T.Hatch: J. Franklin Inst., 215(1933)27-37.

3) T.Itoh, Y.Wanibe and H.Sakao: J. Jpn. Inst. Metals, 49(1985)670-677.

4) T.Itoh and Y.Wanibe: Powder Metallurgy, 34(1991) 126-134.

5) T.Itoh and Y.Wanibe: 5th European Symposium Particle Characterization, K.Leschonski ed., Nuernberg, Euro. Fed. Chem. Eng., NuernbergMesse GmbH, (1992)691-701.

6) T.Itoh and Y.Wanibe: Proceedings of 1993 Powder Metallurgy World Congress, Y.Bando and K.Kosuge ed., Kyoto, Jpn. Soc. Powder and Powder Metallurgy, (1993)319-322.

7) T.Itoh and Y.Wanibe: Advances in Powder Metallurgy \& Particulate Materials-1996 Vol.1, T.M.Cadle and K.S.Narasimhanr ed., Washington D.C., Metal Powder Industries Fed., (1996)Part 4, 41-51.

8) T.Itoh and Y.Wanibe: International Workshop on Modeling of Metal Powder Forming Processes, Grenoble, Organizing Committee, (1997)169-178.

9) T.Itoh and Y.Wanibe: J. Jpn. Soc. Powder and Powder Metallurgy, 41(1994)1111-1116.

10) T.Itoh and Y.Wanibe: Advances in Powder Metallurgy \& Particulate Materials-1994 Vol.2, C.Lall and A.J. Neupaver ed., Toronto, Metal Powder Industries Fed., (1994)113-126.

11) T.Itoh and Y.Wanibe: J. Jpn. Soc. Powder and Powder Metallurgy, 42(1994)992-997. 